\title{
Guest Editorial: Multimedia, Communications, Services and Security
}

\author{
Andrzej Dziech • Andrzej Glowacz • Mikołaj Leszczuk
}

Published online: 26 June 2013

C) The Author(s) 2013. This article is published with open access at Springerlink.com

"Multimedia, Communications, Services and Security" has been an important research topic in recent years and a key part of innovative ICT projects. In particular, numerous intelligent systems benefit from the integration of tools and solutions proposed by scientists, such as image, video and audio processing, development of intelligent sensors, interlinking heterogeneous subsystems with intra- and internet services, and providing reliable and secure communications channels. This special issue consists of eleven papers, which are briefly discussed as follows.

In "Detection and Localization of Selected Acoustic Events in Acoustic Fields for Smart Surveillance Applications," the authors provide a method for determining the position of selected sound events in 3D space. When an event is detected (e.g. speech or impulse sound), the sound source is located so that it can be used in surveillance systems.

The following two papers are related to Quality of Experience issues. "Quality Assessment of Visual and Automatic Licence Plate Recognition" discusses the Quality of Experience in Automatic Licence Plate Recognition tasks based on surveillance videos streamed under constrained networking conditions. Based on the presented assumptions, the authors implement automatic recognition algorithms and compare their accuracy against user perception in order to obtain quality models and critical quality thresholds.

"A Journey from Subjective Psychophysical Experiments to Objective Quality Optimisation" presents two task-based video experiments in surveillance and medical applications. The problem of quality assessment, from subjective psychophysical experiments to objective quality models, is discussed. Example test results and models are provided.

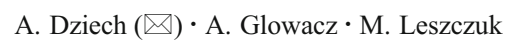
AGH University of Science and Technology, A1. Mickiewicza 30, 30-059 Kraków, Poland e-mail: adzie@tlen.pl
A. Glowacz
e-mail: glowacz@kt.agh.edu.pl
M. Leszczuk
e-mail: leszczuk@kt.agh.edu.pl 
The next two papers are related to digital watermarking of multimedia content. "A New Approach to High-Capacity Annotation Watermarking Based on Digital Fountain Codes" presents a new method of annotation watermarking, based on the fountain coding paradigm. The proposed solution provides high capacity digital watermarks and robustness against JPEG compression or cropping.

In "Chaotic Model-Based Semi-Fragile Watermarking Using Integer Transforms for Digital Fundus Image Authentication," a new reversible, imperceptible, semi-fragile watermarking scheme based on chaotic systems is proposed. The authors show that all mandatory requirements are met, and they evaluate their solution against different watermark attacks.

The paper "INACT-INDECT Advanced Image Cataloguing Tool" sheds some light on the problem of police forces counteracting child pornography and gathering evidence. As a result, fast and accurate tools for cataloguing high- and low-level metadata of evidence are proposed.

The usability of camera systems is the subject of the paper entitled "An InformaticsBased Approach to Object Tracking for Distributed Live Video Computing". The authors extend the Live Video Database Management System prototype with a multifaceted object model to characterize objects in live video streams as a basis for a cross-camera tracking framework. This way, real-time queries may make it possible to monitor complex events and enable rapid application development.

Privacy in high volumes of recorded video is analyzed in " $\mathrm{W}^{3}$-Privacy: Understanding What, When, and Where Inference Channels in Multi-Camera Surveillance Video". A privacy analysis framework is proposed incorporating location, time, activities and other information to determine potential privacy breaches in surveillance systems.

"Analysis of Privacy Vulnerabilities in Single Sign-On Mechanisms for Multimedia Websites" explores privacy risks in OpenID and Facebook Connect. Three solutions to the presented problems are proposed for OpenID.

Biometric features are examined in the following two papers. In "Genetic Programming Extension to APF-Based Monocular Human Body Pose Estimation," the author presents a new method of human body pose estimation. Single 2D camera observations are considered with 3D models of the body and genetic algorithms combined with an annealed particle filter for processing both current and past states of the model.

Finally, "Selection of Parameters in Iris Recognition Systems" presents the analysis of implementation issues occurring during the development of new iris recognition systems. The authors evaluate their solution in terms of performance characteristics and recognition accuracy, verified for the IrisBath database.

The eleven papers listed above cover a wide range of research related to modern multimedia, communications, services and security. We thank all the authors of the submitted papers and all those who have contributed to this special issue of Multimedia Tools and Applications.

Open Access This article is distributed under the terms of the Creative Commons Attribution License which permits any use, distribution, and reproduction in any medium, provided the original author(s) and the source are credited. 


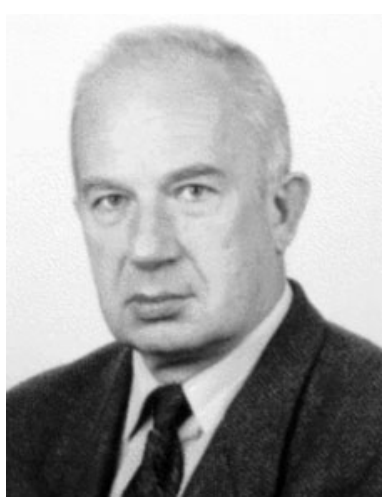

Andrzej Dziech holds the position of a full professor at the Department of Telecommunications of AGH University of Science and Technology in Krakow, Poland. He received his M.Sc. and Ph.D. degrees from the Institute of Electrical Engineering in Saint Petersburg in 1970 and 1973, respectively, and the D.Sc. from Technical University of Poznan in 1978. He is author and co-author of 6 books and approx. 230 publications. He was a supervisor of $18 \mathrm{Ph}$.D.students. His fields of interest are related to digital communication, image and signal processing, data analysis and compression, information and coding theory, random signals, computer communications networks, object recognition and watermarking technology. He was awarded 4 times for research achievements by the Ministry of Education of Poland. Professor Dziech actively participated in numerous international research projects, e.g., Tempus, Knixmas, Calibrate. In addition, he has been coordinator of several large projects as Imcop,Insigma,Tapas. Currently, he is coordinating a European Union FP7 integrated project INDECT.

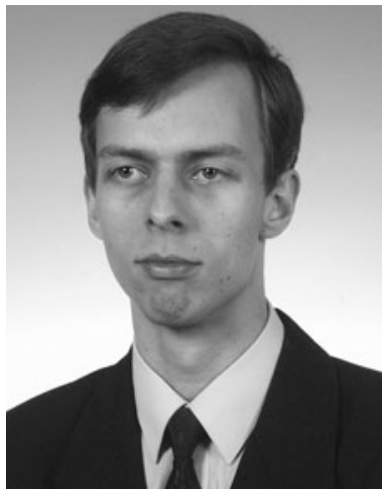

Andrzej Glowacz received his Ph.D. in telecommunications from the AGH University of Science and Technology in 2007. He is currently an Assistant Professor at the AGH University. Andrzej Glowacz has been working on numerous commercial projects, and research projects including INSIGMA (as Deputy Project Coordinator), INTOM (as Deputy Project Coordinator), TAPAS (as Deputy Technology Coordinator), INDECT, GAMA, OASIS Archive, CARMEN, DAIDALOS, DAIDALOS 2, EuroNGI, and EuroFGI. His main professional areas of interest are intelligent information systems, multimedia systems, pattern recognition, wireless QoS, modern transport protocols, and advanced systems programming. He is the author of over 100 scientific papers and technical reports; he also serves as a reviewer of international journals, conferences, and project applications. 


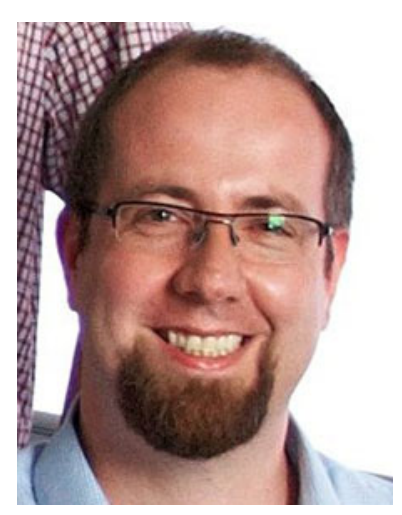

Mikolaj Leszczuk, PhD, is an assistant professor at the Department of Telecommunications, AGH University of Science and Technology (AGH-UST), Krakow, Poland). He received his M.Sc. in Electronics and Telecommunications in 2000 and $\mathrm{PhD}$ degree in Telecommunications in 2006, both from AGH-UST. He is currently teaching Digital Video Libraries, Information Technology and Basics of Telecommunications. In 2000 he visited Universidad Carlos III de Madrid (Madrid, Spain) for a scientific scholarship. During 19971999 he was employed by several Comarch holding companies as a Manager of Research and Development Department, President of the Management and Manager of the Multimedia Technologies Department. He has participated actively as a steering committee member or researcher in several national and European projects, including: INDECT, BRONCHOVID, GAMA, e-Health ERA, PRO-ACCESS, Krakow Centre of Telemedicine, CONTENT, E-NEXT, OASIS Archive, and BTI. He is a member of the VQEG (Video Quality Experts Group) Board and a co-chair of VQEG QART (Quality Assessment for Recognition Tasks) Group. His current activities are focused on e-Health, multimedia for security purposes, P2P, image/video processing (for general purposes as well as for medicine), the development of digital video libraries, particularly video summarization, indexing, compression and streaming subsystems. He has been a chairman of several conference sessions. He is a member of IEEE Society since 2000. He has served as an expert for the European Framework Programme and the Polish State Foresight Programme as well as a reviewer for several conferences publications and journals. 